Bull. Soc. math. France

129 (2), 2001, p. 189-210

\title{
RELATIONS DE FUCHS POUR LES SYSTĖMES DIFFÉRENTIELS RÉGULIERS
}

\author{
PAR EduARdo Corel
}

\begin{abstract}
RÉsumÉ. - Dans cet article, nous montrons que la notion analytique d'exposants développée par Levelt pour les systèmes différentiels linéaires en une singularité régulière s'interprète algébriquement en termes d'invariants de réseaux, relatifs à un réseau stable maximal que nous appelons «réseau de Levelt». Nous obtenons en particulier un encadrement pour la somme des exposants des systèmes n'ayant que des singularités régulières sur $\mathbb{P}^{1}(\mathbb{C})$.

Abstract (Fuchs' relations for regular differential systems). - In this article, we reinterpret A.H.M. Levelt's notion of exponents for linear differential systems at a regular singularity as eigenvalues of the residue of a regular connection on a maximal lattice (that we call "Levelt's lattice"). This allows us to establish upper and lower bounds for the sum of exponents for systems having only regular singularities on $\mathbb{P}^{1}(\mathbb{C})$.
\end{abstract}

La notion d'exposant pour une équation ou un système différentiels linéaires caractérise l'ordre de croissance des solutions en un point singulier régulier. Les résultats contenus dans cet article concernent une généralisation de la relation de Fuchs pour les équations différentielles au cas des systèmes différentiels.

Pour une équation différentielle

$$
y^{(n)}+a_{n-1}(z) y^{(n-1)}+\cdots+a_{0}(z) y=0
$$

Texte reçu le 27 janvier 2000, révisé le 11 juillet 2000

Eduardo Corel, Institut de Mathématiques de Jussieu, 175 rue du Chevaleret, 75013 Paris (France) • E-mail : corel@math.jussieu.fr

Classification mathématique par sujets (2000). — 34A20, 34A30, 12H05, 34C20, 32S40.

Mots clefs. - Système différentiel, point singulier régulier, formes normales, connexion, réseau, exposants, réseau de Levelt, relation de Fuchs. 
fuchsienne sur $\mathbb{P}^{1}(\mathbb{C})$, c'est-à-dire à singularités régulières, la relation de Fuchs (cf. [Po], p. 77) relie les exposants $e_{1}^{s}, \ldots, e_{n}^{s}$, à savoir les ordres maximaux de croissance de $n$ solutions indépendantes, en les points $s \in \mathbb{P}^{1}(\mathbb{C})$, par la formule

$$
\sum_{s \in \mathbb{P}^{1}(\mathbb{C})}\left(\sum_{i=1}^{n} e_{i}^{s}-\frac{1}{2} n(n-1)\right)=-n(n-1) .
$$

Pour un système différentiel d'ordre $n$

$$
\frac{\mathrm{d} X}{\mathrm{~d} z}=A X,
$$

méromorphe sur $\mathbb{P}^{1}(\mathbb{C})$, on dispose, en une singularité régulière $s$, d'une notion d'exposants définie par A.H.M. Levelt [Le1] comme celle des ordres de croissance maximaux $e_{1}^{s}, \ldots, e_{n}^{s}$ d'un système fondamental de solutions du système (1) par rapport à une valuation convenable. On les appellera les exposants de Levelt du système (1) en $s$.

Si les coefficients de la matrice $A$ sont des fractions rationnelles, on définit la hauteur du système (1) comme la somme de ses rangs de Poincaré

$$
h(A)=\sum_{s \in \mathbb{P}^{1}(\mathbb{C})} \sup \left(0,-\operatorname{ord}_{s} A d z-1\right),
$$

où ord ${ }_{s}$ désigne l'ordre d'une fonction, éventuellement à valeurs matricielles. Nous démontrons alors le résultat suivant.

THÉORÈme 1. - Soit $\mathrm{d} X / \mathrm{d} z=A X$ un système différentiel à singularités régulières sur $\mathbb{P}^{1}(\mathbb{C})$, et soient $e_{1}^{s}, \ldots, e_{n}^{s}$ les exposants de Levelt associés à ce système en les points $s \in \mathbb{P}^{1}(\mathbb{C})$. La somme des exposants vérifie alors

$$
-\frac{1}{2} n(n-1) h(A) \leq \sum_{s \in \mathbb{P}^{1}(\mathbb{C})} \sum_{i=1}^{n} e_{i}^{s} \leq-h(A) .
$$

L'inégalité de droite précise un résultat d'A.A. Bolibrukh ([Bo3], prop. 1.2.3, p. 24), qui montre que sous les mêmes hypothèses

$$
\sum_{s \in \mathbb{P}^{1}(\mathbb{C})} \sum_{i=1}^{n} e_{i}^{s} \leq 0 .
$$

Si le système n'a que des pôles simples, on a $h(A)=0$, et l'on retrouve la caractérisation de Bolibrukh des systèmes fuchsiens par la propriété

$$
\sum_{s \in \mathbb{P}^{1}(\mathbb{C})} \sum_{i=1}^{n} e_{i}^{s}=0 .
$$

La double inégalité ci-dessus repose sur une étude algébrique locale des singularités régulières. En notant $K$ le corps local valué $\mathbb{C}((z))$, et $\mathcal{O}$ son anneau

TOME $129-2001-\mathrm{N}^{\mathrm{O}} 2$ 
de valuation $\mathbb{C}[[z]]$, nous parlerons d'un système d'ordre $n$

$$
\frac{\mathrm{d} X}{\mathrm{~d} z}=A X
$$

où $A \in \mathrm{M}_{n}(K)$, comme l'expression dans une base donnée $(e)$ d'un $K$-espace vectoriel à connexion $(V, \nabla)$, de dimension $n$, de l'équation

$$
\nabla_{\mathrm{d} / \mathrm{d} z}(v)=0
$$

où $\nabla_{\mathrm{d} / \mathrm{d} z}$ désigne la contraction de $\nabla$ avec la dérivation usuelle $\mathrm{d} / \mathrm{d} z(c f$. [G-L], p. 164). Les transformations de jauge

$$
A_{[P]}=P^{-1} A P-P^{-1} \frac{\mathrm{d} P}{\mathrm{~d} z},
$$

où $P \in \mathrm{GL}_{n}(K)$ est une matrice non singulière correspondant au changement d'inconnue $X=P Y$, s'interprètent alors comme des changements de base dans $V$. Rechercher une jauge holomorphe $P \in \mathrm{GL}_{n}(\mathcal{O})$ revient à respecter une structure supplémentaire de réseau, c'est-à-dire la structure de $\mathcal{O}$-module engendré par la base $(e)$. Au lieu de caractériser la singularité régulière en termes de croissance modérée des solutions du système, nous nous servirons de l'existence d'un réseau $M$ de $V$ stable sous l'action de l'opérateur $\nabla_{z \mathrm{~d} / \mathrm{d} z}$ (cf. [Ka], p. 211).

À la notion analytique d'exposants de Levelt correspond par cette traduction une notion algébrique, que nous obtenons en utilisant les décompositions suivantes de matrices fondamentales. Si le système différentiel (1), supposé holomorphe en $z=0$, y admet une singularité régulière, il existe une matrice fondamentale $\mathcal{Y}$ de solutions du système sous la forme

$$
\mathcal{Y}=\Omega z^{N} z^{L}
$$

où :

- $\Omega$ est une matrice $n \times n$ non singulière, holomorphe sur un germe de disque $D$ de centre $z=0$;

- $N=\operatorname{diag}\left(N_{1}, \ldots, N_{n}\right)$ est une matrice diagonale d'entiers ordonnés par ordre décroissant;

- $L$ est une matrice constante triangulaire supérieure, dont les valeurs propres appartiennent au sous-ensemble $\{z \in \mathbb{C} \mid \operatorname{Re}(z) \in[0,1[\}$ de $\mathbb{C}$.

En considérant les résidus en 0 dans la relation de Liouville

$$
\frac{\mathrm{d}(\operatorname{det} \mathcal{Y})}{\mathrm{d} z}=(\operatorname{Tr} A) \operatorname{det} \mathcal{Y}
$$

on obtient, à l'aide des décompositions ci-dessus, l'équation

$$
\operatorname{Res}_{0} \operatorname{Tr} A=\operatorname{ord}_{0} \operatorname{det} \Omega+\operatorname{Tr} N+\operatorname{Tr} L .
$$

Le premier à avoir considéré ce type d'écriture est F.R. Gantmacher qui, en 1947 (voir [G], chap. XIV, §10, p. 143 seq.), a montré que, sous l'hypothèse que la matrice $A$ est à pôle simple, on peut supposer que la matrice $\Omega$ est inversible 
à coefficients holomorphes. L'entier $\operatorname{ord} d_{0} \operatorname{det} \Omega$ est alors nul, et les nombres $N_{i}+L_{i i}$ sont les valeurs propres du résidu $A_{-1}$ de la matrice du système.

Dans [Le1], A.H.M. Levelt montre qu'il existe une telle écriture qui vérifie $N_{i}+L_{i i}=e_{i}^{0}$, connue sous le nom de forme normale de Levelt. En remarquant que $\sum_{i=1}^{n} e_{i}^{0}=\operatorname{Tr} N+\operatorname{Tr} L$, on en déduit la relation

$$
\operatorname{Res}_{0} \operatorname{Tr} A=\operatorname{ord}_{0} \operatorname{det} \Omega+\sum_{i=1}^{n} e_{i}^{0} .
$$

On voit alors, par le théorème des résidus, que généraliser la relation de Fuchs revient à évaluer l'ordre $\operatorname{ord}_{s} \operatorname{det} \Omega$ du déterminant de la transformation de jauge $\Omega$ en tout $s \in \mathbb{P}^{1}(\mathbb{C})$.

Nous montrons que le nombre $\operatorname{ord}_{0} \operatorname{det} \Omega$ s'interprète algébriquement comme la dimension $\left[\Lambda: \Lambda_{L}\right]$ du $\mathbb{C}$-espace vectoriel $\Lambda / \Lambda_{L}$, où $\Lambda$ est le réseau engendré par la base $(e)$ dans laquelle on a exprimé le système, et $\Lambda_{L}$ est un autre réseau que nous appelons le réseau de Levelt de $\Lambda$. Cette notion, qui semble dépendre de la matrice $\Omega$, ne dépend en fait que de la classe d'équivalence holomorphe du système.

Le réseau $\Lambda_{L}$ se caractérise algébriquement de la façon très simple suivante.

Proposition. $-\Lambda_{L}$ est le plus grand réseau de $V$ stable sous $\nabla_{z \mathrm{~d} / \mathrm{d} z}$ et contenu dans $\Lambda$.

De ce fait, l'entier $\left[\Lambda: \Lambda_{L}\right]$ est minimal parmi ceux des réseaux stables contenus dans $\Lambda$, et la différence entre $\operatorname{Res}_{0} \operatorname{Tr} A$ et $\sum_{i=1}^{n} e_{i}^{0}$ la plus petite possible.

L'utilité du réseau de Levelt tient au résultat suivant.

THÉORÈme 2. - Les exposants de Levelt du système (1) sont les valeurs propres du résidu de la connexion $\nabla$ pour le réseau de Levelt associé.

Ce résultat constitue la caractérisation algébrique des exposants de Levelt évoquée plus haut, et permet de faire le rapprochement entre la construction de Levelt et les résultats obtenus antérieurement par Gantmacher dans le cas d'un pôle simple.

Dans une première partie, nous rappelons des propriétés algébriques essentielles des réseaux des $K$-espaces vectoriels à connexion. Nous définissons ensuite le réseau de Levelt, dont nous majorons les invariants grâce à la première partie. Nous établissons enfin le théorème 2 ainsi que les inégalités de Fuchs.

\section{Réseaux des espaces vectoriels à connexion}

Pour plus de détails sur les notions que nous présentons dans ce paragraphe, nous renvoyons à $[\mathrm{G}-\mathrm{L}]$.

Soit $K=\mathbb{C}((z))$ le corps des séries méromorphes formelles, muni de la dérivation $\theta=z \mathrm{~d} / \mathrm{d} z$, soient $\mathcal{O}=\mathbb{C}[[z]]$ l'anneau de valuation de $K$ pour la

TOME $129-2001-\mathrm{N}^{\mathrm{O}} 2$ 
valuation $z$-adique $v$, et $\Omega_{K \mid \mathbb{C}}^{1}$ le $K$-espace vectoriel des 1 -formes différentielles sur $\mathbb{C}$.

On désigne par $V$ un $K$-espace vectoriel de dimension finie $n$ muni d'une connexion

$$
\nabla: V \longrightarrow V \otimes_{K} \Omega_{K \mid \mathbb{C}}^{1}
$$

Pour toute $\mathbb{C}$-dérivation $\partial$ de $K$, on note $\nabla_{\partial}$ l'opérateur différentiel de $V$ correspondant

$$
\begin{aligned}
\nabla_{\partial}: V & \longrightarrow V, \\
v & \longmapsto\langle\nabla v, \partial\rangle .
\end{aligned}
$$

On appelle matrice de l'opérateur différentiel $\nabla_{\partial}$ dans une base $(e)$ de $V$, la matrice $A=\left(A_{i, j}\right)$, notée également $\operatorname{Mat}\left(\nabla_{\partial},(e)\right)$, définie par la formule

$$
\nabla_{\partial}\left(e_{j}\right)=-\sum_{i=1}^{n} A_{i, j} e_{i}
$$

pour $j=1, \ldots, n$, où l'on désigne par $e_{i}$ le $i$-ème vecteur de la base $(e)$.

Pour une autre base $(\varepsilon)$ de $V$, la matrice $\operatorname{Mat}\left(\nabla_{\partial},(\varepsilon)\right)=A_{[P]}$ est donnée par la transformation de jauge

$$
A_{[P]}=P^{-1} A P-P^{-1} \partial(P),
$$

où $P \in \mathrm{GL}_{n}(K)$ est la matrice de passage de $(e)$ à $(\varepsilon)$. Un réseau de $V$ est par définition un $\mathcal{O}$-module $\Lambda$ engendré par une base de $V$.

Si $(e)$ est une base de $V$, on note $\mathcal{R}(e)$ le réseau engendré par $(e)$, et l'on dit que $(e)$ est une $(\mathcal{O}$-) base de $\mathcal{R}(e)$.

Lemme 1.1. - Soit $\Lambda$ un réseau de V. Pour tout $K$-automorphisme $\varphi$ de $V$, l'image $\varphi(\Lambda)$ de $\Lambda$ est un réseau, et l'on a $\varphi(\Lambda) \subset \Lambda($ resp. $\varphi(\Lambda)=\Lambda)$ si et seulement s'il existe une base $(e)$ de $\Lambda$ telle que $\operatorname{Mat}(\varphi,(e)) \in \mathrm{M}_{n}(\mathcal{O})$ (resp. $\left.\operatorname{Mat}(\varphi,(e)) \in \mathrm{GL}_{n}(\mathcal{O})\right)$. Cette dernière condition est alors vérifiée pour toute base $(e)$ de $\Lambda$.

Nous rappelons que la connexion $\nabla$ est dite régulière s'il existe un réseau stable sous l'action de $\nabla_{\theta}$.

Lemme 1.2. - Pour tout réseau $\Lambda$ de $V$ les conditions suivantes sont équivalentes :

(i) $\Lambda$ est stable sous l'action de $\nabla_{\theta}$;

(ii) il existe une $\mathcal{O}$-base (e) de $\Lambda$ telle que la matrice $A=\operatorname{Mat}\left(\nabla_{\theta},(e)\right)$ soit à coefficients dans $\mathcal{O}$;

(iii) pour toute $\mathcal{O}$-base $(e)$ de $\Lambda$, la matrice $A=\operatorname{Mat}\left(\nabla_{\theta},(e)\right)$ est à coeffcients dans $\mathcal{O}$.

BULLETIN DE LA SOCIÉtÉ MATHÉMATIQUE DE FRANCE 
1.1. Valuation associée à un réseau. - On rappelle que pour tout réseau $\Lambda$ de $V$, on définit une valuation $v_{\Lambda}$ sur l'espace vectoriel $V$, en posant pour tout $x \in V$

$$
v_{\Lambda}(x)=\sup \left\{k \in \mathbb{Z} \mid x \in z^{k} \Lambda\right\} .
$$

Pour tout réseau $M$ de $V$, et plus généralement pour toute partie non vide $M$ d'un réseau, on pose

$$
v_{\Lambda}(M)=\inf _{x \in M} v_{\Lambda}(x),
$$

en convenant que $v_{\Lambda}(M)=\infty$ si $M=(0)$.

LEMme 1.3. - Soit $\Lambda$ un réseau de $V$.

(i) Pour tout $x \in V$, et toute $\mathcal{O}$-base $(e)$ de $\Lambda$, soit $x=\sum_{i=1}^{n} x_{i} e_{i}$ l'écriture en coordonnées de $x$ dans $(e)$. On a alors

$$
v_{\Lambda}(x)=\min _{i=1, \ldots, n} v\left(x_{i}\right) .
$$

(ii) Pour tout réseau $M$ de $V$, et toute famille $\left(\varepsilon_{1}, \ldots, \varepsilon_{m}\right)$ de $V$ engendrant le réseau $M$ sur $\mathcal{O}$, on a

$$
v_{\Lambda}(M)=\min _{i=1, \ldots, m} v_{\Lambda}\left(\varepsilon_{i}\right)
$$

(iii) La valuation $v_{\Lambda}(M)$ est égale à l'unique entier $k \in \mathbb{Z}$ tel que $z^{-k} M \subset \Lambda$ et $z^{-k-1} M \not \subset \Lambda$.

(iv) Pour tout réseau $M$, on a $v_{\Lambda}\left(z^{-v_{\Lambda}(M)} M\right)=0$.

1.2. Diviseurs élémentaires. — L'anneau $\mathcal{O}$ étant principal, on sait par le théorème des diviseurs élémentaires que si $M$ est un sous-réseau de $\Lambda$, il existe une unique suite croissante d'entiers $k_{1} \leq \cdots \leq k_{n}$ et une base $(e)$ de $\Lambda$ telles que $\left(z^{k_{1}} e_{1}, \ldots, z^{k_{n}} e_{n}\right)$ soit une base de $\bar{M}$.

Dans le cas général, le réseau $z^{-v_{\Lambda}(M)} M$ est un sous-réseau de $\Lambda$. Un réseau de $V$ admet donc toujours une base de la forme ci-dessus.

DÉfinition 1.4. - Soient $\Lambda$ et $M$ deux réseaux de $V$.

(i) On appelle (abusivement) diviseurs élémentaires de $M$ dans $\Lambda$ les entiers

$$
k_{1}=\ell_{1}+v_{\Lambda}(M), \ldots, k_{n}=\ell_{n}+v_{\Lambda}(M)
$$

où $z^{\ell_{1}}, \ldots, z^{\ell_{n}}$ sont les diviseurs élémentaires au sens usuel de $z^{-v_{\Lambda}(M)} M$ dans $\Lambda$.

(ii) On appelle base de Smith de $\Lambda$ pour $M$ toute base (e) de $\Lambda$ telle que la famille $\left(z^{k_{1}} e_{1}, \ldots, z^{k_{n}} e_{n}\right)$ soit une base de $M$.

(iii) On appelle multiplicité d'un diviseur élémentaire $k_{i}$ le nombre $m\left(k_{i}\right)$ d'indices $j$ tels que $k_{j}=k_{i}$. On posera $m(\ell)=0$ si $\ell$ n'est pas un diviseur élémentaire de $M$ dans $\Lambda$.

TOME $129-2001-\mathrm{N}^{\mathrm{O}} 2$ 
Nous écrirons les diviseurs élémentaires de $M$ dans $\Lambda$ sous la forme $k_{i, \Lambda}(M)$ pour préciser si nécessaire les réseaux concernés, et on posera alors

$$
\mathcal{K}_{\Lambda}(M)=\left(k_{1, \Lambda}(M), \ldots, k_{n, \Lambda}(M)\right) .
$$

L'indice d'un sous-réseau $M$ d'un réseau $\Lambda$ de $V$ est défini comme l'entier

$$
[\Lambda: M]=v(\operatorname{det} P)
$$

pour toute matrice $P$ de passage de $\Lambda$ à $M$ (cf. [Mar], p. 14).

Lemme 1.5. - Soient $\Lambda \supset M$ deux réseaux de $V$. On a :

(i) $[\Lambda: M]=\sum_{i=1}^{n} k_{i, \Lambda}(M)$;

(ii) $[\Lambda: M]=\operatorname{dim}_{\mathbb{C}}(\Lambda / M)$.

Proposition 1.6. - Soient $N \subset M$ deux réseaux de $V$, et soit $\Lambda$ un réseau quelconque de $V$. Les diviseurs élémentaires respectifs de $M$ et $N$ dans $\Lambda$ vérifient, pour tout $i=1, \ldots, n$,

$$
k_{i, \Lambda}(M) \leq k_{i, \Lambda}(N) .
$$

Démonstration. - Soit $P$ la matrice de passage d'une base de Smith pour $M$ à une base de Smith pour $N$ dans $\Lambda$. La matrice $z^{-\mathcal{K}_{\Lambda}(M)} P z^{\mathcal{K}_{\Lambda}(N)}$ fait passer d'une base de $M$ à une base de $N$. Par conséquent, d'après le lemme 1.1, on a, pour tout $1 \leq i, j \leq n$,

$$
v\left(P_{i, j} z^{k_{j, \Lambda}(N)-k_{i, \Lambda}(M)}\right) \geq 0 .
$$

Comme $P \in \mathrm{GL}_{n}(\mathcal{O})$, il existe une permutation $\sigma$ telle que $v\left(P_{i, \sigma(i)}\right)=0$ pour tout $i=1, \ldots, n$. D'où la relation $k_{\sigma(i), \Lambda}(N) \geq k_{i, \Lambda}(M)$. Les deux suites étant croissantes, on en déduit que $k_{i, \Lambda}(N) \geq k_{i, \Lambda}(M)$.

1.3. Invariants d'une connexion relatifs à un réseau. - Se donner un réseau $\Lambda$ engendré par une base $(e)$ dans un $K$-espace vectoriel à connexion $\left(V, \nabla_{\partial}\right)$ revient à considérer la classe d'équivalence

$$
\left\{\partial X=A_{[P]} X \mid P \in \mathrm{GL}_{n}(\mathcal{O})\right\}
$$

de systèmes différentiels modulo l'action de jauge $(4)$ de $\mathrm{GL}_{n}(\mathcal{O})$ sur la matrice $A=\operatorname{Mat}\left(\nabla_{\partial},(e)\right)$. À la différence de $\mathrm{GL}_{n}(K)$, l'action de $\mathrm{GL}_{n}(\mathcal{O})$ conserve les quantités suivantes.

1.3.1. Le rang de Poincaré. - Dans la théorie classique, on appelle rang de Poincaré d'un système singulier $\mathrm{d} X / \mathrm{d} z=A X$ le nombre $-v(A)-1$. La définition intrinsèque suivante correspond au système exprimé avec la dérivation $\theta$.

DÉfinition 1.7. - On appelle rang de Poincaré de la connexion $\nabla$ sur le réseau $\Lambda$ l'entier

$$
r(\nabla, \Lambda)=\max \left(-v_{\Lambda}\left(\nabla_{\theta}(\Lambda)\right), 0\right) .
$$

La vérification du lemme suivant est immédiate.

BULLETIN DE LA SOCIÉtÉ MATHÉMATIQUE DE FRANCE 
Lemme 1.8. - Soit $\Lambda$ un réseau de $V$. Le réseau $\Lambda$ est stable sous l'action de $\nabla_{\theta}$ si et seulement si $r(\nabla, \Lambda)=0$.

1.3.2. La trace du résidu. — L'application $\nabla_{\theta}$ induit sur l'espace vectoriel $\wedge^{n} V$ une connexion naturelle, notée $\wedge^{n} \nabla$, qui est régulière. Comme $\operatorname{dim}_{K} \wedge^{n} V=1$, tout réseau de $\wedge^{n} V$ est stable par $\wedge^{n} \nabla$ (cf. [Le2], p. 5). L'application $\wedge^{n} \nabla_{\theta}$ induit donc un endomorphisme $\overline{\wedge^{n}\left(\nabla_{\theta}\right)}$ sur le $\mathbb{C}$-espace vectoriel $\wedge^{n} \Lambda / z \wedge^{n} \Lambda$ de dimension 1.

DÉFINITION 1.9. - On appelle trace du résidu de la connexion $\nabla$ sur le réseau $\Lambda$ le nombre complexe

$$
\tau(\nabla, \Lambda)=\bar{\Lambda}\left(\nabla_{\theta}\right)
$$

Dans une $\mathcal{O}$-base $(e)$ de $\Lambda$, on a $\tau(\nabla, \Lambda)=\operatorname{Tr} \operatorname{Res}_{0} A$, où $A=\operatorname{Mat}\left(\nabla_{\partial},(e)\right)$ est la matrice de la connexion, $T r$ désigne la trace d'une matrice et $\operatorname{Res}_{0}$ sa matrice résidu en $z=0$.

Si la matrice $A$ est à pôle simple, une jauge $P \in \mathrm{GL}_{n}(\mathcal{O})$ la transforme en une matrice à pôle simple $A_{[P]}$ dont le résidu est conjugué du précédent par $P(0)$.

DÉFInition 1.10. - Soit $\Lambda$ un réseau $\nabla_{\theta}$-stable de $V$. On appelle résidu de $\nabla$ sur le réseau $\Lambda$ l'endomorphisme $\operatorname{Res}_{\Lambda} \nabla$ induit sur $\Lambda / z \Lambda$ par la connexion $\nabla_{\theta}$.

Dans une base quotient $\overline{(e)}$ de $\Lambda / z \Lambda$, la matrice de $\operatorname{Res}_{\Lambda} \nabla$ est le résidu de la matrice de la connexion $\nabla_{\mathrm{d} / \mathrm{d} z}$ exprimée dans $(e)$.

1.3.3. Le rang polaire de la connexion. - Pour tout $P \in \mathrm{GL}_{n}(\mathcal{O})$, le terme « le plus polaire » de la matrice $A_{[P]}$ s'obtient en conjuguant le terme le plus polaire de la matrice $A$ par $P(0)$. Si $r>0$ désigne le rang de Poincaré du système (1), on a

$$
\left(A_{[P]}\right)_{-r}=P(0)^{-1} A_{-r} P(0) .
$$

En particulier, le rang de $A_{-r}$ est invariant. Nous l'appellerons le rang polaire de la connexion. Sa définition intrinsèque est la suivante.

DÉfinition 1.11. - Soit $\Lambda$ un réseau de $(V, \nabla)$ et soit $r=r(\nabla, \Lambda)>0$ le rang de Poincaré de $\nabla$ sur $\Lambda$. On appelle rang polaire de la connexion $\nabla$ sur le réseau $\Lambda$ l'entier $\eta(\nabla, \Lambda)$ défini par

$$
\eta(\nabla, \Lambda)=\operatorname{rg}_{\mathbb{C}} \overline{z^{r} \nabla_{\theta}},
$$

où $\overline{z^{r} \nabla_{\theta}}$ désigne l'endomorphisme induit par $z^{r} \nabla_{\theta}$ sur le quotient $\Lambda / z \Lambda$, et $\mathrm{rg}_{\mathbb{C}}$ le rang d'une application linéaire.

TOME $129-2001-\mathrm{N}^{\mathrm{O}} 2$ 


\section{Le réseau de Levelt}

Soit $(V, \nabla)$ un $K$-espace vectoriel de dimension finie à connexion régulière, et $\Lambda$ un réseau de $V$.

Proposition 2.1. - L'ensemble des réseaux stables par $\nabla_{\theta}$ et contenus dans $\Lambda$ admet un unique élément maximal pour l'inclusion.

Démonstration. - $\Lambda$ étant un module de type fini sur l'anneau principal $\mathcal{O}$, il suffit de vérifier le lemme suivant, dont la démonstration est immédiate.

Lemme 2.2. - Soient $M$ et $\widetilde{M}$ deux réseaux de $V$. Le réseau $M+\widetilde{M}$ vérifie les propriétés suivantes:

(i) si $M$ et $\widetilde{M}$ sont $\nabla_{\theta}$-stables, il en est de même pour $M+\widetilde{M}$;

(ii) on a $M+\widetilde{M}=M$ si et seulement si $M \supset \widetilde{M}$.

DÉFinition 2.3. - On appelle réseau de Levelt associé à $\Lambda$, et l'on note $\Lambda_{L}$, le plus grand réseau de $V$ contenu dans $\Lambda$ et stable par $\nabla_{\theta}$.

Proposition 2.4. - Soit $(V, \nabla)$ un $K$-espace vectoriel de dimension finie à connexion régulière et $\Lambda$ un réseau. Le réseau de Levelt $\Lambda_{L}$ associé à $\Lambda$ vérifie les propriétés suivantes:

1) $v_{\Lambda}\left(\Lambda_{L}\right)=0$;

2) pour tout réseau $\nabla_{\theta}$-stable $M \subset \Lambda$, on $a\left[\Lambda: \Lambda_{L}\right] \leq[\Lambda: M]$.

Démonstration. - Le réseau $\Lambda_{L}$ est par définition un sous-réseau de $\Lambda$. On a donc $v_{\Lambda}\left(\Lambda_{L}\right) \geq 0$. D'autre part, si $v_{\Lambda}\left(\Lambda_{L}\right)=k>0$, le réseau $z^{-k} \Lambda_{L}$ est encore stable et contient strictement $\Lambda_{L}$, ce qui contredit la définition de $\Lambda_{L}$, d'où 1). Pour tout sous-réseau $M$ de $\Lambda$, stable sous $\nabla_{\theta}$, on a $M \subset \Lambda_{L}$, donc $k_{i, \Lambda}(M) \geq k_{i, \Lambda}\left(\Lambda_{L}\right)$. Le 2$)$ est alors une conséquence directe des définitions.

Remarquons que si $\Lambda$ est lui-même stable par $\nabla_{\theta}$, on a $\Lambda_{L}=\Lambda$.

\subsection{Invariants de $\nabla$ relatifs à $\Lambda$ : les exposants.}

DÉFINITION 2.5. - On appelle exposants de la connexion régulière $\nabla$ relativement au réseau $\Lambda$, les valeurs propres $e_{i}(\nabla, \Lambda)$ du résidu de la connexion pour le réseau de Levelt associé.

Par définition, le réseau de Levelt de $\Lambda$ est stable par $\nabla_{\theta}$. Dans toute $\mathcal{O}$-base $(\varepsilon)$ de $\Lambda_{L}$, la matrice $A=\operatorname{Mat}\left(\nabla_{\theta},(\varepsilon)\right)$ est à coefficients dans $\mathcal{O}$. Elle s'écrit par conséquent

$$
A=A_{0}+A_{1} z+A_{2} z^{2}+\cdots .
$$

Les exposants de $\nabla$ pour $\Lambda$ sont alors les valeurs propres de la matrice $A_{0} \in \mathrm{M}_{n}(\mathbb{C})$.

Remarque 2.6. - La somme des exposants vaut $\sum_{i=1}^{n} e_{i}(\nabla, \Lambda)=\tau\left(\nabla, \Lambda_{L}\right)$. 
REMARQUE 2.7. - La partie non-entière des exposants est un invariant méromorphe de la connexion. Nous noterons $N_{i}(\nabla, \Lambda)$ les parties entières des parties réelles des exposants $e_{i}(\nabla, \Lambda)$.

2.2. Majoration des invariants du réseau de Levelt. - Le réseau de Levelt est le sous-réseau stable le plus proche de celui de départ au sens de l'indice. Nous déterminons dans ce paragraphe un encadrement de cet indice.

Proposition 2.8. - Soit $(V, \nabla)$ un $K$-espace vectoriel à connexion régulière, $\Lambda$ un réseau de $V$ et $r=r(\nabla, \Lambda)$ le rang de Poincaré de la connexion relativement à $\Lambda$. Soit $\Lambda_{L}$ le réseau de Levelt associé. Les diviseurs élémentaires $0=k_{1} \leq \cdots \leq k_{n}$ de $\Lambda_{L}$ dans $\Lambda$ vérifient la double inégalité

$$
\max _{i=1, \ldots, n-1}\left(k_{i+1}-k_{i}\right) \leq r \leq k_{n} .
$$

Démonstration. - Si $r=0$, on a $\Lambda_{L}=\Lambda$, et donc $k_{1}=\cdots=k_{n}=0$. Nous supposerons dans la suite que $r>0$.

Soit $(\varepsilon)$ une base de Smith de $\Lambda$ pour $\Lambda_{L}$. Nous noterons $X=\left(x_{1}, \ldots, x_{n}\right)$ un $n$-uplet d'entiers, $z^{X}$ la matrice

$$
z^{X}=\left(\begin{array}{ccc}
z^{x_{1}} & & 0 \\
& \ddots & \\
0 & & z^{x_{n}}
\end{array}\right)
$$

et $\left(z^{X} \varepsilon\right)$ la famille $\left(z^{x_{1}} \varepsilon_{1}, \ldots, z^{x_{n}} \varepsilon_{n}\right)$. La matrice de la connexion dans la base $(\varepsilon)$ étant notée $A=\operatorname{Mat}\left(\nabla_{\theta},(\varepsilon)\right)$, on a

$$
\operatorname{Mat}\left(\nabla_{\theta},\left(z^{X} \varepsilon\right)\right)=A_{\left[z^{X}\right]}=\left(A_{i, j} z^{x_{j}-x_{i}}-\delta_{i, j} x_{i}\right)_{1 \leq i, j \leq n} .
$$

Le réseau de Levelt $\Lambda_{L}$ est stable sous $\nabla_{\theta}$. La matrice $A_{\left[z^{\mathcal{K}}\right]}$ avec $\mathcal{K}=\left(k_{1}, \ldots, k_{n}\right)$ est donc à coefficients dans $\mathcal{O}$. On a ainsi

$$
v\left(A_{i, j}\right)-k_{i}+k_{j} \geq 0 \quad \text { pour } \quad 1 \leq i, j \leq n .
$$

Comme $r=\max _{1 \leq i, j \leq n}\left(-v\left(A_{i, j}\right)\right)$, on obtient l'inégalité de droite

$$
r \leq \max _{1 \leq i, j \leq n}\left(k_{j}-k_{i}\right)=k_{n} .
$$

D'autre part, l'indice $\left[\Lambda: \Lambda_{L}\right]=\sum_{i=1}^{n} k_{i}$ est minimal parmi les indices dans $\Lambda$ des sous-réseaux stables de $\Lambda$. Pour tout $T=\left(t_{1}, \ldots, t_{n}\right) \in \mathbb{Z}^{n}$ tel que

$$
0 \leq t_{1} \leq \cdots \leq t_{n} \quad \text { et } \quad \sum_{i=1}^{n} t_{i}<\sum_{i=1}^{n} k_{i}
$$

le réseau engendré par $\left(z^{T} \varepsilon\right)$ n'est pas $\nabla_{\theta}$-stable. Il existe donc un couple d'indices $\left(i_{(T)}, j_{(T)}\right) \in\{1, \ldots, n\}^{2}$ tels que

$$
v\left(A_{i_{(T)}, j_{(T)}}\right)-t_{i_{(T)}}+t_{j_{(T)}}<0 .
$$

TOME $129-2001-\mathrm{N}^{\mathrm{O}} 2$ 
Soit $\ell$ tel que $k_{\ell+1} \geq 1$. Montrons que

$$
k_{\ell+1}-k_{\ell} \leq r .
$$

On pose $t_{i}=k_{i}$ pour $i \leq \ell$ et $t_{i}=k_{i}-1$ pour $i \geq \ell+1$. Il existe alors un couple $(i, j)$ et $\varepsilon=-1,0$ ou 1 tels que

$$
0>v\left(A_{i, j}\right)-t_{i}+t_{j}=v\left(A_{i, j}\right)-k_{i}+k_{j}+\varepsilon \geq \varepsilon .
$$

On a donc $v\left(A_{i, j}\right)=k_{i}-k_{j}$ et $i \leq \ell \leq \ell+1 \leq j$. On en déduit que

$$
k_{\ell+1}-k_{\ell} \leq k_{j}-k_{i}=-v\left(A_{i, j}\right) \leq r .
$$

L'inégalité de gauche $\max _{i=1, \ldots, n-1} k_{i+1}-k_{i} \leq r$ s'ensuit.

Corollaire 2.9. - Soit $\Lambda$ un réseau de $V$.

1) Les diviseurs élémentaires du réseau de Levelt $\Lambda_{L}$ dans $\Lambda$ vérifient

$$
k_{i, \Lambda}\left(\Lambda_{L}\right) \leq(i-1) r
$$

pour tout $i=1, \ldots, n$.

2) L'indice du réseau de Levelt $\Lambda_{L}$ dans $\Lambda$ vérifie les inégalités

$$
r \leq\left[\Lambda: \Lambda_{L}\right] \leq \frac{1}{2} n(n-1) r,
$$

où $r$ désigne le rang de Poincaré de $\nabla$ sur $\Lambda$.

Proposition 2.10. - Soit $\Lambda$ un réseau instable de $V$. Si le rang de polarité vérifie $\eta(\nabla, \Lambda)=n-1$, on a

$$
\left[\Lambda: \Lambda_{L}\right]=\frac{1}{2} n(n-1) r .
$$

Les diviseurs élémentaires sont dans ce cas

$$
\mathcal{K}_{\Lambda}\left(\Lambda_{L}\right)=(0, r, 2 r, \ldots,(n-1) r) .
$$

Démonstration. - Soit $(e)$ une base de Smith de $\Lambda$ pour $\Lambda_{L}$, et

$$
A=\operatorname{Mat}\left(\nabla_{\theta},(e)\right) \text {. }
$$

D'après (5), la matrice $A_{-r}$ est triangulaire supérieure de diagonale nulle, et sa première colonne est également nulle. Si $\operatorname{rg} A_{-r}=n-1$, la «sur-diagonale »

$$
\left(A_{i, i+1}^{(-r)}\right)_{i=1}^{n-1}
$$

ne peut alors contenir aucun zéro. Par conséquent, on a $k_{i+1}-k_{i} \geq r$ pour tout $i=1, \ldots, n-1$. La proposition 2.8 entraîne alors l'égalité $k_{i+1}-k_{i}=r$.

À l'opposé de ce cas où l'indice est maximal, on a le résultat suivant. 
Corollaire 2.11. - Avec les mêmes notations, s'il existe un sousréseau stable $M$ de $\Lambda$ dont les diviseurs élémentaires dans $\Lambda$ vérifient $\mathcal{K}_{\Lambda}(M)=(0,0, \ldots, 0, r)$, alors on a

$$
M=\Lambda_{L} .
$$

REMARQUE 2.12. - Si la dimension de $V$ est $n=2$, on a nécessairement $\left[\Lambda: \Lambda_{L}\right]=r$ et $\mathcal{K}_{\Lambda}\left(\Lambda_{L}\right)=(0, r)$.

\section{Exposants}

La définition des exposants de Levelt, que nous rappelons plus en détail au 3.1 ci-dessous, est formulée dans le cadre analytique. Nous considérons donc ici le corps $k=\mathbb{C}\{z\}\left[z^{-1}\right]$ des séries entières convergentes méromorphes en 0 . Le corps $k$ est un sous-corps différentiel du corps $K=\mathbb{C}((z))$. L'étude classique des systèmes analytiques à singularité régulière, par exemple dans $[\mathrm{G}]$ ou $[\mathrm{Wa}]$, montre que les objets introduits dans les parties précédentes sont également analytiques. En particulier, il en résulte l'existence du réseau de Levelt obtenu en remplaçant l'anneau $\mathcal{O}=\mathbb{C}[[z]]$ par l'anneau $R=\mathbb{C}\{z\}$ des séries entières convergentes en $z=0$ (qui est l'anneau de valuation de $k$ correspondant à la restriction de la valuation $v$ ).

3.1. Rappel de la définition des exposants de Levelt. - Nous suivons ici en grande partie la présentation d'A. Beauville [Be].

Considérons un système différentiel d'ordre $n$

$$
\frac{\mathrm{d} X}{\mathrm{~d} z}=A X
$$

holomorphe sur une couronne $D^{*}=\{z \in \mathbb{C}|0<| z \mid<\rho\}$, où $\rho>0$ est un réel arbitraire, et admettant un point singulier régulier en $z=0$.

Soient $p: \widetilde{D}^{*} \rightarrow D^{*}$ un revêtement universel de $D^{*}$ et $\log z$ une détermination du logarithme sur $D^{*}$. Pour toute matrice $C \in \mathrm{GL}_{n}(\mathbb{C})$, on note

$$
\log C
$$

l'unique matrice de $\mathrm{M}_{n}(\mathbb{C})$ telle que $\mathrm{e}^{\log C}=C$ et telle que ses valeurs propres soient éléments de l'ensemble $\{z \in \mathbb{C} \mid \operatorname{Im}(z) \in[0,2 \pi[\}$. Une fonction holomorphe sur $\widetilde{D}^{*}$ est dite à croissance modérée s'il existe un réel $\lambda$ tel que, pour toute section holomorphe locale $s$ de $p$, on ait

$$
\lim _{z \rightarrow 0} z^{-\lambda} f(s(z))=0 .
$$

On note $\mathcal{H}_{\text {mod }}\left(\widetilde{D}^{*}\right)$ l'espace des fonctions holomorphes sur $\widetilde{D}^{*}$ à croissance modérée, et l'on pose, pour $f \in \mathcal{H}_{\bmod }\left(\widetilde{D}^{*}\right)$,

$$
\hat{v}(f)=\sup \left\{k \in \mathbb{Z} \mid \forall \lambda<k, \forall s \text { section de } p, \lim _{z \rightarrow 0} z^{-\lambda} f(s(z))=0\right\} .
$$

TOME $129-2001-\mathrm{N}^{\mathrm{O}} 2$ 
Comme le système (7) est à singularité régulière en $z=0$, l'espace $\mathcal{X}$ des solutions de $(7)$ est un sous- $\mathbb{C}$-espace vectoriel de $\left(\mathcal{H}_{\bmod }\left(\widetilde{D}^{*}\right)\right)^{n}$. On étend la valuation $\hat{v}$ à l'espace $\mathcal{X}$ en posant

$$
\begin{aligned}
& \hat{v}: \mathcal{X} \longrightarrow \mathbb{Z}, \\
& y=\left(f_{i}\right)_{i=1, \ldots, n} \longmapsto \hat{v}(y)=\min _{i=1, \ldots, n} \hat{v}\left(f_{i}\right) .
\end{aligned}
$$

Les ensembles $\mathcal{X}_{k}=\{y \in \mathcal{X} \mid \hat{v}(y) \geq k\}$ forment une filtration décroissante de $\mathcal{X}$. Considérons la suite des entiers $N_{1} \geq \cdots \geq N_{n}$, obtenue en répétant $\operatorname{dim}_{\mathbb{C}}\left(\mathcal{X}_{i} / \mathcal{X}_{i+1}\right)$ fois l'entier $i$ quand $i$ parcourt $\mathbb{Z}$. Il existe une base $(y)$ de $\mathcal{X}$ telle que $\hat{v}\left(y_{i}\right)=N_{i}$ pour tout $i=1, \ldots, n$ et telle que la matrice $G$ de la monodromie du système exprimée dans $(y)$ soit triangulaire supérieure $(c f$. [Be], p. 7). On pose

$$
L=\frac{1}{2 i \pi} \log G
$$

DÉfinition 3.1. - Avec les notations précédentes, on appelle valuations de Levelt du système (7) les nombres $N_{1}, \ldots, N_{n}$ et exposants de Levelt du système (7) les nombres $N_{1}+L_{11}, \ldots, N_{n}+L_{n n}$.

On vérifie que cette définition ne dépend pas de la base $(y)$ choisie.

La suite $N_{1}, \ldots, N_{n}$ représente les maxima successifs de l'application $\hat{v}$ (voir [Le1], p. 375). En particulier, si l'on note $\mathcal{Y}_{i}$ la $i$-ème colonne d'une matrice fondamentale $\mathcal{Y}$ de solutions de $(7)$, et si $\hat{v}\left(\mathcal{Y}_{1}\right) \geq \cdots \geq \hat{v}\left(\mathcal{Y}_{n}\right)$, alors $\hat{v}\left(\mathcal{Y}_{i}\right) \leq N_{i}$ pour tout $i=1, \ldots, n$ (cf. [Be], p. 7). Nous notons $N$ la matrice diagonale $\operatorname{diag}\left(N_{1}, \ldots, N_{n}\right)$

Le but de cette partie est d'établir le résultat suivant.

THÉORÈme 3.2. - Les exposants de Levelt du système (7) sont égaux aux valeurs propres du résidu (c'est-à-dire aux exposants au sens de la définition 2.5), de la connexion $\nabla$ définie par (7) dans la base canonique de $K^{n}$, relativement au réseau canonique $\mathcal{O}^{n}$.

La démonstration de ce théorème s'appuie sur la proposition 3.3 ci-dessous.

Soit $\mathcal{M}$ l'ensemble des triplets $(\Omega, T, L)$, que nous appellerons dans la suite triplets GLB (pour Gantmacher-Levelt-Bolibrukh), où

- $\Omega$ est une matrice $n \times n$ non singulière, holomorphe sur le disque $D=D^{*} \cup\{0\}$

- $T=\operatorname{diag}\left(T_{1}, \ldots, T_{n}\right)$ est une matrice diagonale à coefficients dans $\mathbb{Z}$;

- $L$ est une matrice constante dont les valeurs propres appartiennent au sous-ensemble $\{z \in \mathbb{C} \mid \operatorname{Re}(z) \in[0,1[\}$ de $\mathbb{C}$.

BULLETIN DE LA SOCIÉtÉ MATHÉMATIQUE DE FRANCE 
En notant $\mathcal{F}$ l'anneau des fonctions holomorphes sur $\widetilde{D}^{*}$, on associe à tout triplet $(\Omega, T, L)$ le produit $\Omega z^{T} z^{L}$ dans l'anneau $M_{n}(\mathcal{F})$ des matrices d'ordre $n$ à coefficients dans $\mathcal{F}$, où l'on note

$$
z^{L}=\mathrm{e}^{L \log z}
$$

On appelle matrice fondamentale de solutions de $(7)$ (relativement à $\mathcal{F}$ ) tout élément $\mathcal{Y} \in \mathrm{GL}_{n}(\mathcal{F})$ tel que $\mathrm{d} \mathcal{Y} / \mathrm{d} z=A \mathcal{Y}$. On dit qu'une matrice fondamentale $\mathcal{Y}$ admet une factorisation $G L B$ s'il existe un triplet $\operatorname{GLB}(\Omega, T, L)$ tel que $\mathcal{Y}=\Omega z^{T} z^{L}$.

Définissons les ensembles $\mathcal{M}^{\text {faible }}$, composé des triplets GLB tels que $z^{T} L z^{-T}$ soit holomorphe, et $\mathcal{M}^{\text {fort }}$, composé des triplets GLB pour lesquels $T_{1} \geq \cdots \geq T_{n}$ et $L$ est triangulaire supérieure, ainsi que leurs sous-ensembles respectifs $\mathcal{M}_{0}^{\text {faible }}$ et $\mathcal{M}_{0}^{\text {fort }}$ pour lesquels on impose de plus que $\Omega(0)$ soit une matrice inversible. Remarquons que $\mathcal{M}^{\text {fort }} \subset \mathcal{M}^{\text {faible }}$ et que $\mathcal{M}_{0}^{\text {fort }} \subset \mathcal{M}_{0}^{\text {faible }}$.

Nous rappelons, et rassemblons dans la proposition suivante, trois résultats essentiels concernant les «formes normales » et la matrice $N$ des valuations de Levelt.

Proposition 3.3. - Avec les notations ci-dessus, on a :

(i) Il existe $(\Omega, T, L) \in \mathcal{M}^{\text {fort }}$, où $T=N$, tel que $\Omega z^{T} z^{L}$ soit une matrice fondamentale de solutions du système (7) (cf. [Bo3], p. 16). Une telle écriture s'appelle forme normale de Levelt.

(ii) Si la matrice $A$ admet en $z=0$ au plus un pôle simple (c'est-à-dire $\left.\operatorname{ord}_{0} A \geq-1\right)$, alors il existe $(\Omega, T, L) \in \mathcal{M}_{0}^{\text {fort }}$, tel que $\Omega z^{T} z^{L}$ soit une matrice fondamentale de solutions du système (7). De plus, les nombres $T_{i}+L_{i i}$ sont les valeurs propres de la matrice résidu de $A$ (cf. [G], ch. XIV, $\S 10$, th. 2, p.157). Nous appelons une telle écriture forme normale de Gantmacher.

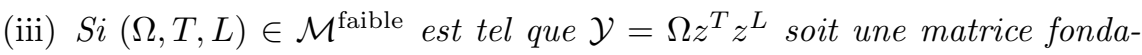
mentale de solutions du système $(7)$, on a $\hat{v}\left(\mathcal{Y}_{i}\right) \geq T_{i}$ pour tout $i=1, \ldots, n$ (cf. [Bo3], cor. 1.2.1, p. 21).

Démonstration du théorème 3.2. — L'application «développement de Taylor» permet de considérer $A$ comme une matrice à coefficients dans le corps $K=$ $\mathbb{C}((z))$, complété formel du corps $k=\mathbb{C}\{z\}\left[z^{-1}\right]$, le système (7) définissant une connexion régulière $\nabla$ sur $K^{n}$. Le réseau de Levelt $\left(\mathcal{O}^{n}\right)_{L}$ associé à $\nabla$ et au réseau canonique $\mathcal{O}^{n}$ de $K^{n}$ est en fait défini sur l'anneau de valuation $R$ de $k$, c'est-à-dire $\left(\mathcal{O}^{n}\right)_{L}=\left(R^{n}\right)_{L} \otimes_{k} K$, où $\left(R^{n}\right)_{L}$ est le réseau de Levelt associé à $R^{n}$ (voir [Ma], prop. 1.2). Il existe donc une matrice $W$ holomorphe et inversible dans $M_{n}(k)$, qui est la matrice de passage de la base canonique de $k^{n}$ à une base du réseau de Levelt $\left(R^{n}\right)_{L}$, et telle que le système

$$
\frac{\mathrm{d} Z}{\mathrm{~d} z}=B Z
$$

TOME $129-2001-\mathrm{N}^{\mathrm{O}} 2$ 
de matrice $B=W^{-1} A W-W^{-1} \mathrm{~d} W / \mathrm{d} z$ soit à pôle simple.

D'après la proposition 3.3, il existe une matrice fondamentale $\mathcal{Y}=\Omega z^{N} z^{L}$ de $(7)$ où $(\Omega, N, L) \in \mathcal{M}^{\text {fort }}$, et une matrice fondamentale $\tilde{\mathcal{Z}}=\tilde{\Omega} z^{\widetilde{N}} z^{\tilde{L}}$ de $(8)$ où $(\tilde{\Omega}, \tilde{N}, \tilde{L})$ appartient à $\mathcal{M}_{0}^{\text {fort }}$. Alors les $N_{i}+L_{i i}$ sont les exposants de Levelt (voir [Bo3], p. 16), et d'après la prop. 3.3 (ii), les $\widetilde{N}_{i}+\tilde{L}_{i i}$ sont les exposants de la définition 2.5.

Comme $\tilde{\mathcal{Y}}=W \tilde{\mathcal{Z}}$ est également une matrice fondamentale de (7), il existe une matrice $C \in \mathrm{GL}_{n}(\mathbb{C})$ telle que $W \tilde{\mathcal{Z}}=\mathcal{Y} C$. On en déduit que

$$
W \tilde{\Omega} z^{\tilde{N}} z^{\tilde{L}}=\Omega z^{N} z^{L} C=\Omega z^{N} C z^{C^{-1} L C} .
$$

Dans ces conditions, $\mathrm{e}^{2 i \pi L}$ est la matrice de monodromie de $\mathcal{Y}$, et $\mathrm{e}^{2 i \pi \tilde{L}}$ celle de $\tilde{\mathcal{Z}}$, donc aussi de $W \tilde{\mathcal{Z}}=\mathcal{Y} C$. On a ainsi $\tilde{L}=C^{-1} L C$, et $W \tilde{\Omega} z^{\tilde{N}}=\Omega z^{N} C$. En prenant la valuation des déterminants de ces matrices, on obtient la relation

$$
v(\operatorname{det} W)+v(\operatorname{det} \tilde{\Omega})+\operatorname{Tr} \widetilde{N}=v(\operatorname{det} \Omega)+\operatorname{Tr} N .
$$

La matrice $\tilde{\Omega}$ est inversible dans $M_{n}(R)$, d'où $v(\operatorname{det} \tilde{\Omega})=0$, et

$$
\operatorname{Tr} \widetilde{N}-\operatorname{Tr} N=v(\operatorname{det} \Omega)-v(\operatorname{det} W) .
$$

Comme $(\Omega, N, L) \in \mathcal{M}^{\text {fort }}$, la matrice $\Omega$ fait passer dans $k^{n}$ de la base canonique à une base d'un réseau stable $\mathcal{N}$ de $k^{n}$ inclus dans le réseau canonique $R^{n}$. Le réseau de Levelt étant maximal, on a $\left[R^{n}: \mathcal{N}\right]=v(\operatorname{det} \Omega) \geq v(\operatorname{det} W)$, d'où l'on déduit que $\operatorname{Tr} \widetilde{N} \geq \operatorname{Tr} N$.

D'autre part, comme $(\tilde{\Omega}, \tilde{N}, \tilde{L}) \in \mathcal{M}_{0}^{\text {fort }}$, on a $(W \tilde{\Omega}, \tilde{N}, \tilde{L}) \in \mathcal{M}^{\text {fort }}$. D'après la proposition 3.3 (iii), les colonnes $\tilde{\mathcal{Y}}_{i}$ de $\tilde{\mathcal{Y}}$ vérifient $\hat{v}\left(\tilde{\mathcal{Y}}_{i}\right) \geq \widetilde{N}_{i}$ pour tout $i=1, \ldots, n$. Soit alors $\sigma$ une permutation qui réordonne les $\hat{v}\left(\tilde{\mathcal{Y}}_{i}\right)$ par ordre décroissant, de sorte que $\hat{v}\left(\tilde{\mathcal{Y}}_{i}\right) \leq N_{\sigma^{-1}(i)}$. En sommant terme à terme, on obtient l'inégalité

$$
\sum_{i=1}^{n} N_{\sigma^{-1}(i)} \geq \sum_{i=1}^{n} N_{i}
$$

On en déduit que $\operatorname{Tr} \widetilde{N}=\operatorname{Tr} N$. Les deux suites étant décroissantes, on a $\widetilde{N}_{i}=N_{i}$ pour tout $i=1, \ldots, n$.

Par conséquent, le réseau $\mathcal{N}$ donné par $\Omega$ est égal au réseau $\left(R^{n}\right)_{L}$ de Levelt, donc $z^{N} C z^{-N}=\Omega^{-1} W \tilde{\Omega} \in \mathrm{GL}_{n}(R)$. On montre alors que l'action de la matrice $C$ sur $L$ permute les valeurs propres de $L$ correspondant à des éléments égaux de $N$, d'où l'égalité

$$
N_{i}+L_{i i}=\tilde{N}_{i}+\tilde{L}_{i i} \text { pour tout } i=1, \ldots, n
$$

qui achève la preuve du théorème 3.2 .

Dans une forme normale de Levelt $\Omega z^{N} z^{L}$, la jauge $\Omega$ est une matrice de passage de la base d'origine du système à une base du réseau de Levelt associé 
à ce système. À l'aide des résultats de la partie 2.2 , on peut majorer a priori l'écart entre le système de départ et le système à pôle simple le plus proche. Ces majorations conduisent à des estimations sur la somme des exposants.

\section{Inégalités de Fuchs}

Dans ce paragraphe, nous prendrons pour corps $K$ le corps des fractions rationnelles $\mathbb{C}(z)$, muni en tout point $a \in \mathbb{P}^{1}(\mathbb{C})$ de l'application de valuation locale $\operatorname{ord}_{a}$. On note

$$
\operatorname{ord}_{a} A=\min _{1 \leq i, j \leq n} \operatorname{ord}_{a} A_{i, j}
$$

l'ordre en $a$ d'une matrice $A$, et $\operatorname{Res}_{z=a} f$ le résidu d'une fonction $f(z)$ au point $z=a$. En un point $s \in \mathbb{P}^{1}(\mathbb{C})$, les définitions locales précédentes s'étendent en effectuant le changement de coordonnée $t=z-s$ si $s \in \mathbb{C}$ et $t=1 / z$ si $s=\infty$. On désignera en particulier le rang de Poincaré au point $s$ par $r_{s}$.

DÉFInition 4.1. - Si la matrice $A$ est à coefficients dans $\mathbb{C}(z)$, on appelle hauteur du système $\mathrm{d} X / \mathrm{d} z=A X$ l'entier

$$
h(A)=\sum_{a \in \mathbb{P}^{1}(\mathbb{C})} \sup \left(0,-\operatorname{ord}_{a} A \mathrm{~d} z-1\right) .
$$

LEMME 4.2. - Pour tout système (1) à coefficients dans $\mathbb{C}(z)$, on a :

(i) $h(A)=\sum_{s \in \mathbb{P}^{1}(\mathbb{C})} r_{s}$;

(ii) $h(A)=0$ si et seulement s'il existe $a_{1}, \ldots, a_{p}$ dans $\mathbb{C}$ et $A_{1}, \ldots, A_{p}$ dans $\mathrm{M}_{n}(\mathbb{C})$ tels que $A=\sum_{i=1}^{p} A_{i} /\left(z-a_{i}\right)$.

Démonstration. - Le (i) se déduit aisément de la définition de la hauteur. Si $h(A)=0$, la forme différentielle $A \mathrm{~d} z$ est au plus à pôles simples sur $\mathbb{P}^{1}(\mathbb{C})$. Le (ii) en découle directement (voir par exemple [Bo3], p. 1).

ThÉORÈme 4.3 (relation de Fuchs). - Soit $\mathrm{d} X / \mathrm{d} z=A X$ un système différentiel à singularités régulières sur $\mathbb{P}^{1}(\mathbb{C})$. Les exposants de Levelt $e_{1}^{s}, \ldots, e_{n}^{s}$ associés à ce système en les points $s \in \mathbb{P}^{1}(\mathbb{C})$ vérifient

$$
-\frac{1}{2} n(n-1) h(A) \leq \sum_{s \in \mathbb{P}^{1}(\mathbb{C})} \sum_{i=1}^{n} e_{i}^{s} \leq-h(A) .
$$

REMARQUE 4.4. - Si le système est d'ordre $n=2$, l'encadrement ci-dessus devient l'égalité $\sum_{s \in \mathbb{P}^{1}(\mathbb{C})}\left(e_{1}^{s}+e_{2}^{s}\right)=-h(A)$.

Démonstration du théorème 4.3. - On se ramène à $z=0$ en utilisant une coordonnée locale. Le système (1) définit une connexion $\nabla$ sur $K^{n}$. On associe à $\mathcal{O}^{n}$ son réseau de Levelt $\left(\mathcal{O}^{n}\right)_{L}$. Pour toute matrice $P$ faisant passer de la

TOME $129-2001-\mathrm{N}^{\mathrm{O}} 2$ 
base canonique de $K^{n}$ à une $\mathcal{O}$-base de $\left(\mathcal{O}^{n}\right)_{L}$, la matrice $A_{[P]}$ est à pôle simple. Par le théorème 3.2 , on a

$$
\begin{aligned}
\sum_{i=1}^{n} e_{i}^{0} & =\operatorname{Res}_{0} \operatorname{Tr} A_{[P]}=\operatorname{Res}_{0} \operatorname{Tr}\left(P^{-1} A P-P^{-1} \frac{\mathrm{d} P}{\mathrm{~d} z}\right) \\
& =\operatorname{Res}_{0} \operatorname{Tr} A-v(\operatorname{det} P)=\operatorname{Res}_{0} \operatorname{Tr} A-\left[\Lambda: \Lambda_{L}\right] .
\end{aligned}
$$

Le corollaire 2.9 entraîne que

$$
\operatorname{Res}_{t=0} \operatorname{Tr} A-\frac{1}{2} n(n-1) r_{0} \leq \sum_{i=1}^{n} e_{i}^{0} \leq \operatorname{Res}_{t=0} \operatorname{Tr} A-r_{0} .
$$

En sommant sur toutes les singularités, on obtient

$$
\begin{aligned}
\sum_{s \in \mathbb{P}^{1}(\mathbb{C})} \operatorname{Res}_{t=s} \operatorname{Tr} A-\frac{1}{2} n(n-1) & \sum_{s \in \mathbb{P}^{1}(\mathbb{C})} r_{s} \\
& \leq \sum_{s \in \mathbb{P}^{1}(\mathbb{C})} \sum_{i=1}^{n} e_{i}^{s} \leq \sum_{s \in \mathbb{P}^{1}(\mathbb{C})}{\underset{t=s}{\operatorname{Res}} \operatorname{Tr} A-\sum_{s \in \mathbb{P}^{1}(\mathbb{C})} r_{s} .}^{\text {Res }}
\end{aligned}
$$

Le théorème des résidus et le lemme 4.2 terminent la preuve.

\section{Comportement par dualité}

Étant donné un espace vectoriel à connexion $(V, \nabla)$, il existe une connexion canonique $\nabla^{*}$ induite par $\nabla$ sur le $K$-dual $V^{*}$ de $V$ (on peut définir une connexion canonique pour toute construction de $V, c f .[\mathrm{M}])$. Elle est définie pour toute $\mathbb{C}$-dérivation $\partial$ de $K$ par la formule, pour tout $f \in V^{*}$, et tout $v \in V$

$$
\left(\nabla_{\partial}^{*}(f)\right)(v)=\partial(f(v))-f\left(\nabla_{\partial}(v)\right) .
$$

La dualité s'étend aux réseaux de façon convenable ( $c f$. [Bou], VII, $\S 4, n^{\circ} 2$, p. 243).

Lemme 5.1. - Soit $M$ un réseau de $V$ engendré sur $\mathcal{O}$ par la base $(e)$ et soit $\left(e^{*}\right)$ la base duale de $(e)$. On a alors

$$
\operatorname{Hom}_{K}(M, \mathcal{O})=\operatorname{Hom}_{\mathcal{O}}(M, \mathcal{O})=\mathcal{R}\left(e^{*}\right) .
$$

On note ce réseau $M^{*}$ et on l'appelle réseau dual de $M$.

Si la connexion $\nabla$ s'exprime dans $(e)$ comme le système différentiel

$$
\frac{\mathrm{d} X}{\mathrm{~d} z}=A X
$$

alors la connexion duale s'exprime dans $\left(e^{*}\right)$ par le système

$$
\frac{\mathrm{d} X}{\mathrm{~d} z}=-{ }^{t} A X .
$$

BULLETIN DE LA SOCIÉTÉ MATHÉMATIQUE DE FRANCE 
Le rang de Poincaré du dual $M^{*}$ est donc $r\left(\nabla^{*}, M^{*}\right)=r(\nabla, M)$. Si $M$ est un sous-réseau de $\Lambda$ et $M$, on a, au contraire, $M^{*} \supset \Lambda^{*}$. On est donc amené à poser la définition suivante, qui, d'après la proposition 2.1, a bien un sens.

Définition 5.2. - Soit $\Lambda$ un réseau de $V$. On appelle co-réseau de Levelt associé à $\Lambda$, et l'on note $\Lambda^{L}$, le plus petit réseau stable contenant $\Lambda$.

Il s'agit de la notion duale de la définition 2.3.

Proposition 5.3. - Soit $\Lambda$ un réseau de $V$. Soient $\left(k_{1}, \ldots, k_{n}\right)$ les diviseurs élémentaires du réseau de Levelt $\Lambda_{L}$ dans $\Lambda$ et $\left(k_{1}^{*}, \ldots, k_{n}^{*}\right)$ ceux du réseau de Levelt $\left(\Lambda^{*}\right)_{L}$ dans $\Lambda^{*}$. On a alors :

1) $\left(\Lambda^{*}\right)_{L}=\left(\Lambda^{L}\right)^{*}$;

2) $k_{i}+k_{n+1-i}^{*} \leq k_{n}$ pour tout $i=1, \ldots, n$; en particulier $k_{n}^{*}=k_{n}$.

Démonstration. - Le 1) est une conséquence immédiate des définitions.

2) Le réseau $\left(\Lambda_{L}\right)^{*}$ est $\nabla_{\theta}^{*}$-stable et, par hypothèse, ses diviseurs élémentaires relatifs à $\Lambda^{*}$ sont $\left(-k_{n}, \ldots,-k_{1}\right)$. Sa valuation dans $\Lambda^{*}$ est donc $v_{\Lambda^{*}}\left(\left(\Lambda_{L}\right)^{*}\right)=-k_{n}$. Comme le réseau $\left(\Lambda^{*}\right)_{L}$ est maximal dans $V^{*}$, on obtient l'inclusion

$$
z^{k_{n}}\left(\Lambda_{L}\right)^{*} \subset\left(\Lambda^{*}\right)_{L}
$$

La proposition 1.6 entraîne que $k_{i, \Lambda^{*}}\left(z^{k_{n}}\left(\Lambda_{L}\right)^{*}\right) \geq k_{i}^{*}$. Or on a

$$
k_{i, \Lambda^{*}}\left(z^{k_{n}}\left(\Lambda_{L}\right)^{*}\right)=k_{n}-k_{n+1-i},
$$

ce qui montre 2). Les réseaux $\Lambda_{L}$ et $\left(\Lambda^{*}\right)_{L}$ sont des réseaux de Levelt, donc on a $k_{1}=k_{1}^{*}=0$. Par conséquent, $k_{n}=k_{n}^{*}$.

Corollaire 5.4. - Les diviseurs élémentaires du réseau de Levelt du dual de $\Lambda$ dans $\Lambda^{*}$ sont les diviseurs élémentaires de $\Lambda$ dans le co-réseau de Levelt $\Lambda^{L}$ de $\Lambda$. Autrement dit, $\mathcal{K}_{\Lambda^{*}}\left(\left(\Lambda^{*}\right)_{L}\right)=\mathcal{K}_{\Lambda^{L}}(\Lambda)$.

\section{Réseau de Levelt et réseau saturé de Gérard-Levelt}

Dans le but de déterminer di vers invariants d'irrégularité, Gérard et Levelt, dans [G-L], définissent en 1973 la notion de réseau saturé.

Reprenons les notations du chapitre 1 . Soit $\Lambda$ un réseau de $V$, et soit $\vartheta$ une $\mathbb{C}$-dérivation de $K$. Pour tout $k \in \mathbb{N}$, on appelle $k$-ième saturé de $\Lambda$ pour la dérivation $\vartheta$ le réseau

$$
\mathcal{F}_{\vartheta}^{k}(\Lambda)=\Lambda+\nabla_{\vartheta}(\Lambda)+\cdots+\nabla_{\vartheta}^{k}(\Lambda) .
$$

Quand la connexion est régulière, on associe ainsi à tout réseau un réseau stable, de façon canonique.

TOME $129-2001-\mathrm{N}^{\mathrm{O}} 2$ 
ThÉORÈme 6.1 (Gérard-Levelt). — Si la connexion $\nabla$ est régulière, alors pour tout réseau $\Lambda$ de $V$, le $(n-1)$-ième saturé $\mathcal{F}_{\theta}^{n-1}(\Lambda)$ de $\Lambda$ est $\nabla_{\theta}$-stable. Nous le notons $\mathcal{F}(\Lambda)$ et l'appelons saturé de Gérard-Levelt de $\Lambda$ (pour $\nabla_{\theta}$ ).

Le réseau saturé encadre les réseaux que nous avons définis. En effet, on a

$$
z^{-v_{\Lambda}(\mathcal{F}(\Lambda))} \mathcal{F}(\Lambda) \subset \Lambda_{L} \subset \Lambda \subset \Lambda^{L} \subset \mathcal{F}(\Lambda) .
$$

Dans [Cor2], appendice, nous avons utilisé ce résultat pour obtenir des approximations des exposants de Levelt. Depuis ce travail, A.H.M. Levelt a obtenu un algorithme général pour déterminer le réseau de Levelt d'un réseau quelconque (cf. [Le4]). À partir d'un sous-réseau stable du réseau $\Lambda$ de départ (typiquement on prend $z^{-v_{\Lambda}(\mathcal{F}(\Lambda))} \mathcal{F}(\Lambda)$ ), il construit un sous-réseau stable de $\Lambda$ qui est strictement plus grand tant que l'on n'a pas atteint le réseau $\Lambda_{L}$.

On peut néanmoins conclure sans algorithme dans un cas particulier.

Proposition 6.2. - Si le rang polaire de la connexion $\nabla$ vérifie

alors on a

$$
\eta(\nabla, \Lambda)=n-1
$$

$$
\Lambda_{L}=z^{(n-1) r} \mathcal{F}(\Lambda) \text {. }
$$

Remarquons que pour $n=2$, on a sans aucune hypothèse $\Lambda_{L}=z^{r} \mathcal{F}(\Lambda)$.

Démonstration. - Si $r=0$, on a $\Lambda=\Lambda_{L}=\mathcal{F}(\Lambda)$. Nous supposerons que l'on a $r>0$. On procède en deux étapes.

1) On montre que $v_{\Lambda}(\mathcal{F}(\Lambda))=-(n-1) r$. Soit $(e)$ une base de $\Lambda$. On note

$$
A=\operatorname{Mat}\left(\nabla_{\theta},(e)\right)=A_{-r} z^{-r}+\cdots+A_{-1} \frac{1}{z}+A_{0}+\cdots
$$

Dans la base $(e)$, le réseau $\mathcal{F}(\Lambda)$ est engendré par les colonnes d'une matrice de format $n \times n^{2}(c f$. [Le3]) donnée par

$$
\mathcal{M}_{\mathcal{F}}(\nabla,(e))=\mathcal{M}_{\mathcal{F}}(A)=\left(I \mathcal{A A}_{2} \ldots \mathcal{A}_{n-1}\right),
$$

où l'on a posé

$$
\mathcal{A}_{0}=I, \quad \mathcal{A}_{k+1}=\theta \mathcal{A}_{k}+A \mathcal{A}_{k} \quad \text { pour tout } k \geq 0 .
$$

Les termes « les plus polaires » de la matrice élargie $\mathcal{M}_{\mathcal{F}}(A)$ proviennent de colonnes de la matrice $\left(A_{-r}\right)^{n-1}$. La matrice $A_{-r}$ est nilpotente de rang $\operatorname{rg} A_{-r}=\eta(\nabla, \Lambda)=n-1$. La matrice $\left(A_{-r}\right)^{n-1}$ est donc non nulle. Il existe par conséquent un vecteur dans $\mathcal{F}(\Lambda)$ dont la valuation par rapport à $\Lambda$ est exactement $-(n-1) r$.

2) Les diviseurs élémentaires de $\Lambda_{L}$ sont, d'après le lemme 2.10,

$$
\mathcal{K}_{\Lambda}\left(\Lambda_{L}\right)=(0, r, \ldots,(n-1) r) .
$$

Comme le rang polaire du dual est $\eta\left(\nabla^{*}, \Lambda^{*}\right)=\eta(\nabla, \Lambda)$, on a de même

$$
\mathcal{K}_{\Lambda^{*}}\left(\left(\Lambda^{*}\right)_{L}\right)=(0, r, \ldots,(n-1) r) .
$$

BULLETIN DE LA SOCIÉtÉ MATHÉMATIQUE DE FRANCE 
Posons $t_{i}=k_{i, \Lambda}(\mathcal{F}(\Lambda))$ pour tout $i=1, \ldots, n$. Comme $\Lambda^{L} \subset \mathcal{F}(\Lambda)$, on a

$$
k_{i, \Lambda}(\mathcal{F}(\Lambda)) \leq k_{i, \Lambda}\left(\Lambda^{L}\right)
$$

pour tout $i=1, \ldots, n$. Or $k_{i, \Lambda}\left(\Lambda^{L}\right)=-k_{n+1-i}^{*}=-(n-i) r$, d'où l'on déduit

$$
t_{i} \leq-(n-i) r .
$$

pour tout $i=1, \ldots, n$.

Inversement, l'inclusion $z^{(n-1) r} \mathcal{F}(\Lambda) \subset \Lambda_{L}$ entraîne que

$$
k_{i, \Lambda}\left(z^{(n-1) r} \mathcal{F}(\Lambda)\right) \geq k_{i, \Lambda}\left(\Lambda_{L}\right) .
$$

On a donc $(n-1) r+t_{i} \geq k_{i, \Lambda}\left(\Lambda_{L}\right)=(i-1) r$, d'où pour tout $i=1, \ldots, n$,

$$
t_{i} \geq-(n-i) r \text {. }
$$

De $(13)$ et $(14)$, on déduit que $t_{i}=-(n-i) r$ pour tout $i=1, \ldots, n$. On a alors

$$
\left[\Lambda: z^{(n-1) r} \mathcal{F}(\Lambda)\right]=\sum_{i=1}^{n}((n-1) r-(n-i) r)=\frac{1}{2} n(n-1) r=\left[\Lambda: \Lambda_{L}\right] .
$$

Par conséquent, on a $\Lambda_{L}=z^{(n-1) r} \mathcal{F}(\Lambda)$. Remarquons également que $\Lambda^{L}=\mathcal{F}(\Lambda)$.

\section{BIBLIOGRAPHIE}

[A-B] Anosov (D.V.), Bolibrukh (A.A.) - The Riemann-Hilbert problem, Aspects of Math., E22, Vieweg \& Sohn, Braunschweig, 1994.

[B-V] BabBitt (D.G.), VARAdarajan (V.S.) - Formal reduction theory of meromorphic differential equations : a group theoretic view, Pacif. J. Math., t. 109, 1983, p. 1-80.

[B-J-L] Balser (W.), Jurkat (W.B.), Lutz (D.A.) - A General Theory of Invariants for Meromorphic Differential Equations, Part I : Formal Invariants, Funkcialaj Ekvacioj, t. 22, 1979, p. 197-221.

[Be] Beauville (A.) - Le problème de Riemann-Hilbert (d'après A.A. Bolibrukh), Sém. Bourbaki, Astérisque 216, 1993, p. 103-119.

[B-L] Bertrand (D.), LAumon (G.) - Appendice à Exposants, vecteurs cycliques et majorations de mutiplicités, C.R. conf. franco-japonaise, Preprint IRMA, Strasbourg, 1985.

[Bi] Birkhoff (G.D.) - Singular points of ordinary linear differential equations, Trans. Amer. Soc., t. 10 1909, p. 436-470.

[Bo1] Bolibrukh (A.A.) - The Riemann-Hilbert problem, Russian Math. Surveys, t. 45, 1990, p. 1-58.

TOME $129-2001-\mathrm{N}^{\mathrm{O}} 2$ 
[Bo2] BolibrukH (A.A.) - Construction of a Fuchsian Equation from a Monodromy Representation, Math. Notes Ac. Sci. of USSR, t. 48-5, 1990, p. 1090-1099.

[Bo3] Bolibrukh (A.A.) - The 21 $1^{\text {st }}$ Hilbert problem for Linear Fuchsian Systems, Proc. Steklov Inst. Math., Vol. 206-5, Amer. Math. Soc., 1995.

[Bou] Bourbaki (N.) - Algèbre Commutative, chap. 5-7, Masson, 1985.

[Co] Cohen (H.) - A Course in Computational Algebraic Number Theory, GTM 138, Springer-Verlag, 1991.

[Cor] COREL (E.) - Inégalités de Fuchs pour les systèmes différentiels réguliers, C.R. Acad. Sci. Paris, t. 328, Série I, 1999, p. 983-986.

[Cor2] CoRel (E.) - Exposants, réseaux de Levelt et relations de Fuchs pour les systèmes différentiels réguliers, Thèse Doctorat $3^{\mathrm{e}}$ cycle, Univ. Louis Pasteur, Strasbourg, 1999.

[Dek] DeKkeRs (W.J.M.) - The matrix of a connection having regular singularities on a vector bundle of rank 2 on $\mathbb{P}^{1}(\mathbb{C})$, Équations différentielles et systèmes de Pfaff dans le champ complexe, Lect. Notes in Math. 712, Springer-Verlag, 1979, p. 33-43.

[De] Deligne (P.) - Équations différentielles à points singuliers réguliers, Lect. Notes in Math. 163, Springer-Verlag, 1970.

[G] Gantmacher (F.R.) - Théorie des matrices, vol. 2 (Questions spéciales et applications), Dunod, Paris, 1966.

[G-L] GÉRARD (R.), LEVELT (A.H.M.) - Invariants mesurant l'irrégularité en un point singulier d'un système d'équations différentielles linéaires, Ann. Inst. Fourier, Grenoble, t. 23-1, 1973, p. 157-195.

[Ka] Katz (N.) - Nilpotent connections and the monodromy theorem. Applications of a result of Turrittin, Publ. Math. IHES, t. 39, 1970, p. 176232.

[Le1] Levelt (A.H.M.) - Hypergeometric functions, II, Nederl. Akad. Wetensch., Proc. Ser. A, t. 64, 1961, p. 373-385.

[Le2] LeVelt (A.H.M.) - Jordan decomposition for a class of singular differential operators, Arkiv för matematik, t. 13, 1975, p. 1-27.

[Le3] Levelt (A.H.M.) - Stabilizing differential operators. A method for computing invariants at irregular singularities, Comput. Math. Appl., Academic Press, London, 1991.

[Le4] Levelt (A.H.M.) - Appendice à l'article d'Eduardo Corel : Calcul des réseaux de Levelt, Bull. Soc. Math. France, t. 129 (2), 2001, p. 211-213.

[Ma] Malgrange (B.) - Connexions méromorphes, II : le réseau canonique, Invent. Math. t. 124, 1996, p. 367-387.

[M] Manin (Y.) - Moduli fuchsiani, Ann. Sc. Norm. Sup. Pisa, t. 19-1, Serie III, 1965, p. 113-126. 
[Mar] Martinet (J.) - Les réseaux parfaits des espaces euclidiens, Masson, 1996.

[Po] Poole (E.G.C.) - Introduction to the theory of linear differential equations, Dover, New-York, 1960.

[Sa1] SABBAH (C.) - Introduction to the algebraic theory of linear systems of differential equations, in Éléments de la théorie des systèmes différentiels, D-modules cohérents et holonomes, Les cours du CIMPA, Coll. Travaux en cours, Hermann, 1993, p. 1-80.

[Sa2] SABbah (C.) - Déformations isomonodromiques et variétés de Frobenius. Une introduction, preprint École Polytechnique, 2000.

[Wa] Wasow (W.) - Asymptotic expansions for ordinary differential equations, Interscience, New York, 1965. 